\title{
Optic Nerve Sheath Ultrasound: Where do We Go from Here?
}

\author{
Kishore Pichamuthu(
}

Indian Journal of Critical Care Medicine (2021): 10.5005/jp-journals-10071-23795

Raised intracranial pressure (ICP) is a common complication in neurocritical care patients. Identifying raised ICP promptly and monitoring changes in ICP through the course of the patient's illness are vital to ensuring good clinical outcomes. Various procedures and interventions unrelated to the brain have also been recognized to increase ICP. Clinical signs are woefully inadequate for diagnosing and tracking raised ICP. Traditional imaging techniques, such as computed tomography $(\mathrm{CT})$ and magnetic resonance imaging (MRI) require transport of the patient, may involve radiation, and are not frequently repeatable. They may not be available in secondary hospital setups in our country. Invasive ICP measurement is continuous and accurate but has its drawbacks of invasiveness: procedural skill requirement, infection, and hemorrhage. Ultrasound evaluation of the diameter of the optic nerve sheath visualized behind the globe with a high-frequency ultrasound probe has been recognized to be a good way to detect and track raised ICP.

The anatomical basis of this ultrasound imaging is the fact that the meningeal layers of the brain continue around the optic nerve up to the globe of the eye. The dural and arachnoid layers fuse to form the optic nerve sheath. The subarachnoid cerebrospinal fluid (CSF) space around the optic nerve is therefore in continuity with the CSF in the chiasmatic cistern. An increase in CSF pressure leads to distension of the dura-arachnoid sheath and an increase in its diameter. Fibrous trabeculae between the pia and the arachnoid ensure that the sheath is tightly bound to the pia for most of the length of the nerve with only a potential CSF space. ${ }^{1,2}$ At the termination of the optic nerve, where it meets the globe, the trabeculae are more elastic so they allow distension of the optic nerve sheath. Thus, the optic nerve sheath distends in response to an elevated CSF pressure only from its attachment to the sclera up to $6-8 \mathrm{~mm}$ behind the globe. ${ }^{3}$ The point of maximum distension is about $3 \mathrm{~mm}$ posterior to the globe.

Optic nerve sheath ultrasound can be performed in patients who are supine or with the head-end elevated up to $30^{\circ}$ with a head neutral position. The optic nerve is insonated through the globe by placing a high-frequency linear probe over the closed eyelid. Images are acquired in axial, parasagittal, and infraorbital coronal planes. The optic nerve is visualized as an anechoic stripe posterior to the sclera. The sheath appears as a hyperechoic layer on either side of the nerve. The optic nerve sheath diameter (ONSD) is measured using calipers from the inner surface of the sheath $3 \mathrm{~mm}$ behind the globe. Measurements are taken in different planes and then averaged.

Although this ultrasound tool was described in $1987^{4}$ and expanded on in $1996^{5}$, it began to be widely used only after studies were published in $2006 .{ }^{6}$ Since then there have been a plethora of publications delving into various aspects of optic nerve ultrasound. Each of these looks into one of three domains in ONSD research. First, there are several articles looking to emphasize the positive correlation between ONSD by ultrasound and raised ICP, detected
Division of Critical Care, Christian Medical College, Vellore, Tamil Nadu, India

Corresponding Author: Kishore Pichamuthu, Division of Critical Care, Christian Medical College, Vellore, Tamil Nadu, India, Phone: +91 9894428858, e-mail: drpichamuthu@yahoo.com

How to cite this article: Pichamuthu K. Optic Nerve Sheath Ultrasound: Where do We Go from Here? Indian J Crit Care Med 2021;25(4):360-361.

Source of support: Nil

Conflict of interest: None

either by invasive measurements ${ }^{7}$ or by traditional neuroimaging such as CT and MRI. ${ }^{8}$ Meta-analyses of studies done in this domain demonstrate very high sensitivity, specificity, positive and negative predictive values for ONSD in diagnosing of raised ICP. The second domain of focus of ONSD research lies in looking to expand the range of applications of ONSD. The range of applications now includes preeclampsia, high altitude mountain sickness, idiopathic intracranial hypertension, head injury, acute liver failure, and ventriculoperitoneal shunt obstruction. ${ }^{9,10}$ The last domain of research focus has been to show how ONSD can change rapidly in response to rapid changes in ICP, thus allowing for real-time tracking of ICP in response to thecal infusions, lumbar puncture, CSF drainage, and procedures such as endotracheal suctioning. ${ }^{11}$

Contributing to this last area of research, Kapoor et al. in the current issue of IJCCM have studied the changes in ONSD that occur during various stages of percutaneous tracheostomy in neurocritical care patients. ${ }^{12}$ These authors have shown that the ONSD rises during all stages of percutaneous tracheostomy, though only five patients had a rise significant enough to warrant osmotherapy. It is important to emphasize here that the differences in measured ONSD between various phases of the procedure are insignificant, below the minimum detectable difference (the differences are very small and are smaller than the magnitude of inter and intraobserver variation), with significant overlap of standard deviations. This means that the differences in ONSD during various stages of the percutaneous tracheostomy were insignificant in the majority of the studied patients. There is truly little information on whether optic nerve sheath US can be used to monitor ICP during procedures on neurocritical care patients and the current study attempts to fill the wide gap. An important takeaway from this study is that it is feasible to monitor ONSD at the bedside during procedures in the intensive care unit (ICU).

If the evidence so strongly suggests that ultrasound ONSD reliably diagnoses and tracks raised ICP, why is it not being used more widely in day-to-day intensive care practice? One of the most common reasons is the inter- and intraobserver variation that we encounter, making it 
less reliable and reproducible at the bedside. The second reason is like all other ultrasound modalities, ONSD is time and labor-intensive.

I believe that to rid us all of our disillusionment with ONSD and ensure that patients benefit from this revolutionary way of noninvasively monitoring ICP, we need to have a four-pronged approach.

The first approach is to standardize the procedure. We know that variations in probe frequency, view, gain, and placement of cursors all have an impact on the measured ONSD. ${ }^{13}$ We need a consensus on the correct procedural technique and that points to the grade the quality of the image. We need to do this at the earliest. This will also reduce the heterogeneity of ONSD research, which impairs applicability.

The second step is to stop being obsessed with numbers. A lot of unreliability of ONSD stems from the uncertainty around the correct placement of the cursors to measure the diameter, especially in patients without raised ICP. Moving to a qualitative, pattern recognition-based approach to diagnose raised ICP is reliable and more reproducible. ${ }^{13}$ This qualitative approach with the incorporation of papilledema greatly increases the ease of diagnosing raised ICP by a novice. Quantitative ONSD measurements can be reserved for patients with raised ICP to track changes over time.

The last step is to ensure adequate training prior to performing ONSD scans. While the number of scans needed to train an ultrasound expert has been determined to be 10, novices may need 25 scans before independent practice. The training needs to focus on elucidating the anatomical details of the nerve-sheath-globe complex. Particular attention needs to be focused on training in identifying possible artifacts, including lamina cribrosa associated edge artifacts, and using retinal artery Doppler to identify the correct position of the optic nerve. ${ }^{14}$

Finally, we need more studies that can compare ONSD-based treatment regimens with standard treatment to determine if ONSD ultrasound results in the improvement of meaningful clinical outcomes.

Urgent steps along these lines will ensure that this unique window into the brain has its correct and well-deserved place at the bedside of a patient with raised ICP.

\section{OrCID}

Kishore Pichamuthu @ http://orcid.org/000-0002-6977-1183

\section{References}

1. Hansen $\mathrm{HC}$, Helmke K. The subarachnoid space surrounding the optic nerves. An ultrasound study of the optic nerve sheath. Surg Radiol Anat 1996;18:323-328. DOI: 10.1007/BF01627611.

2. Killer HE, Laeng HR, Flammer J, Groscurth P. Architecture of arachnoid trabeculae, pillars, and septa in the subarachnoid space of the human optic nerve: anatomy and clinical considerations. $\mathrm{Br} \mathrm{J}$ Ophthalmol 2003;87(6):777-781. DOI: 10.1136/bjo.87.6.777.

3. Pichamuthu KK, Prithishkumar IJ. Appearance of the optic nerve sheath diameter (ONSD) using higher frequency linear probes in detection and monitoring of raised intracranial pressures: a cadaveric study. J Clin Diagn Res 2019;13(7):AC05-AC08. DOI: 10.7860/ JCDR/2019/36359.12973.

4. Gangemi M, Cennamo G, Maiuri F, D'Andrea F. Echographic measurement of the optic nerve in patients with intracranial hypertension. Neurochirurgia (Stuttg) 1987;30(2):53-55. DOI: 10.1055/s-2008-1053656.

5. Helmke K, Hansen HC. Fundamentals of transorbital sonographic evaluation of optic nerve sheath expansion under intracranial hypertension. I. Experimental study. Pediatr Radiol 1996;26(10):701705. DOI: $10.1007 /$ BF01383383.

6. Geeraerts T, Merceron S, Benhamou D, Vigué B, Durantea J. Noninvasive assessment of intracranial pressure using ocular sonography in neurocritical care patients. Intensive Care Med 2008;34(11):20622067. DOI: 10.1007/s00134-008-1149-x.

7. Dubourg J, Javouhey E, Geeraerts T, Messerer M, Kassai B. Ultrasonography of optic nerve sheath diameter for detection of raised intracranial pressure: a systematic review and meta-analysis. Intensive Care Med 2011;37(7):1059-1068. DOI: 10.1007/s00134-0112224-2.

8. Ohle R, Mclsaac SM, Woo MY, Perry JJ. Sonography of the optic nerve sheath diameter for detection of raised intracranial pressure compared to computed tomography. J Ultrasound Med 2015;34(7):1285-1294. DOI: 10.7863/ultra.34.7.1285.

9. Dubost C, Le Gouez A, Jouffroy V, Roger-Christoph S, Benhamou D, Mercier FJ, et al. Optic nerve sheath diameter used as ultrasonographic assessment of the incidence of raised intracranial pressure in preeclampsia: a pilot study. Anesthesiology 2012;116:1066-1071. DOI: 10.1097/ALN.0b013e318246ea1a.

10. Sutherland Al, Morris DS, Owen CG, Bron AJ, Roach RC. Optic nerve sheath diameter, intracranial pressure and acute mountain sickness on Mount Everest: a longitudinal cohort study. Br J Sports Med 2008;42(3):183-188. DOI: 10.1136/bjsm.2007.045286. PMID: 18182624.

11. Maissan IM, Dirven PJ, Haitsma IK, Hoeks SE, Gommers D, Stolker RJ. Ultrasonographic measured optic nerve sheath diameter as an accurate and quick monitor for changes in intracranial pressure. J Neurosurg 2015;123(3):743-747. DOI: 10.3171/2014.10.JNS141197.

12. Kapoor I, Wanchoo J, Mahajan C, Singhal V, Roy H, Kumar S, et al. Effect of percutaneous tracheostomy on optic nerve sheath diameter [TONS Trial]. Indian J Crit Care Med 2021;25(4):382-387.

13. Pichamuthu K, Peter JV, Pritishkumar IJ, Samuel P, Joseph M, John G. Impact of variations in the measurement of optic nerve sheath diameter on the diagnosis of raised intracranial pressure: an appraisal of quantitative and qualitative methods. (S186) in ESICM 2010 MONDAY SESSIONS 11 October 2010. Intensive Care Med 2010;36:86-204. DOI: 10.1007/s00134-010-1999-x.

14. Copetti R, Cattarossi L. Optic nerve ultrasound: artifacts and real images. Intensive Care Med 2009;35(8):1488-1489; author reply 1490-1491. DOI: 10.1007/s00134-009-1494-4. 